Copyright (C) 2014 IEEE. Personal use of this material is permitted. Permission from IEEE must be obtained for all other uses, in any current or future media, including reprinting/republishing this material for advertising or promotional purposes, creating new collective works, for resale or redistribution to servers or lists, or reuse of any copyrighted component of this work in other works. 


\title{
A Methodology to find Influential Prosumers in Prosumer Community Groups
}

\author{
A.J. Dinusha Rathnayaka, Vidyasagar M. Potdar, Tharam Dillon, Omar Hussain, and Elizabeth Chang
}

\begin{abstract}
Smart-grid has created an emerging entity of "prosumer" in energy value network, who not only consumes energy but also generates and shares the green energy with the utility grid. Hence, effective management of prosumers has become pivotal to ensure long-term, sustainable energy sharing process. Recently, the concept of Prosumer Community Group (PCG) has emerged as one of the most promising and effective ways to manage prosumers. However, developing sustainable PCGs is challenging. One of the key challenges in this regard is to assess the contribution made by individual prosumers of a PCG, and find a sub-set of the most influential prosumers whose behaviour would facilitate the long-term sustainability of the PCG. In this research paper, we have focused on this challenge and proposed an innovative methodology to assess and rank the prosumers, in order to build an influential membership base. We have assessed the long-term and short-term energy behaviours of prosumers based on a multiple evaluation criteria, and accordingly decided the ranks of the prosumers, whereby the higher ranked prosumers are deemed to be more influential in enhancing the long-term sustenance of the PCG. Furthermore, we have presented simulation results to verify our proposed methodology. The current literature on smart-grid research field has no work investigating this challenge, making our contribution novel.
\end{abstract}

Index Terms-Prosumer, smart-grid, energy sharing

\section{INTRODUCTION}

$\mathrm{T}$ HE demand for energy in the world is continually rising. Worldwide energy consumption is expected to increase by 49 percent from 2007 to $2035[1]$ and the majority of this current demand is met by non-renewable energy sources. However, the society is now faced with dwindling scarce nonrenewable energy resources, thus, the conventional energy users are encouraged to generate and consume the renewable energy and feed the surplus back to the grid[2-4]. In order to achieve such a bi-directional energy and information flow between the energy users and the utility grid, the vision of smart grid has been proposed in literature [5]. Smart grid (SG) refers to "an electricity grid that can intelligently integrate the behavior and actions of all users connected to it in order to deliver sustainable, economic and secure electricity supplies" [5].

The smart grid has introduced a new role of 'prosumer' in energy network, where a "prosumer" is defined as an energy user, who generates renewable energy in its domestic environment (such as roof-top solar systems) and shares the

Copyright (c) 2009 IEEE. Personal use of this material is permitted. However, permission to use this material for any other purposes must be obtained from the IEEE by sending a request to pubs-permissions@iee.org surplus with the energy buyers such as utility grid[6, 7].

Building automation is one of the key requisites to accomplish the integration of prosumers into the utility grid[8]. For instance, various energy consuming loads and domestic distributed energy resources (DER) are connected to each other via a Home Area Network (HAN). HANs are further linked to the local power distribution network through Smart Metering Infrastructure (SMI), forming a local area network where the prosumer communicates with power companies[9].

Recently, a massive number of energy consumers have initiated green energy sharing with the utility grid[5]. However as a single entity, such prosumer is not in a position to produce and sell enough electricity to have a bargaining power in the competitive energy market. Even if the prosumers are grouped in an ad-hoc manner to attain higher quantity of energy, there is perceived uncertainty that the group members may supply whatever amount of energy they prefer, resulting in unreliable energy sharing in the longterm[7].

However, if the prosumers are grouped on the basis of their relatively similar energy sharing behaviours, and are inspired to achieve a common goal (such as, to produce sufficient quantity of energy that would enable a higher feed-in tariff), they can be formed as an opt-in sort of Prosumer Community Groups (PCG). Accordingly, we define the term 'Prosumer Community Group' (PCG) as "a network of prosumers, having relatively similar energy sharing behaviours, who endeavour to pursue a mutual goal and jointly compete in the energy market"[10]. The key advantage of this approach is that it reliably increases the accumulated quantity of energy to be auctioned or sold in the energy market, thus attaining a relatively higher bargaining power compared to single prosumers or ad-hoc prosumer groups, and thus establishing a more sustainable energy sharing process. As a result, the small players (prosumers) receive the negotiating power to be at par with big players (providers) and eliminate the dividing gap between them. Such community-based energy sharing network is particularly advantageous in the remote areas that do not have abundant energy sources, and where huge costs and transmission difficulties are involved in transporting energy to satisfy the energy needs of the inhabitants. In such situations, a strong interaction between the prosumers, consumers and the utility-grid will induce each individual to work together to manage their electricity usage more efficiently.

In such community based energy sharing network, a PCG interacts with each other and with the utility grid through the community gateway as shown in Fig.1. The community 
gateway refers to a smart intermediary that bridges the utility grid with smart gateways of each distributed energy resource that belongs to a community group, in order to achieve collective goals based on community group's interests and policies. The smart storage, on the other hand, represents a physical storage facility that can temporarily store unused electricity, which can then be utilized to offload the energy consumption of the prosumers of the same PCG, as required. Such a community based energy sharing network can be managed by a central operating platform, called Community Management Platform[7].

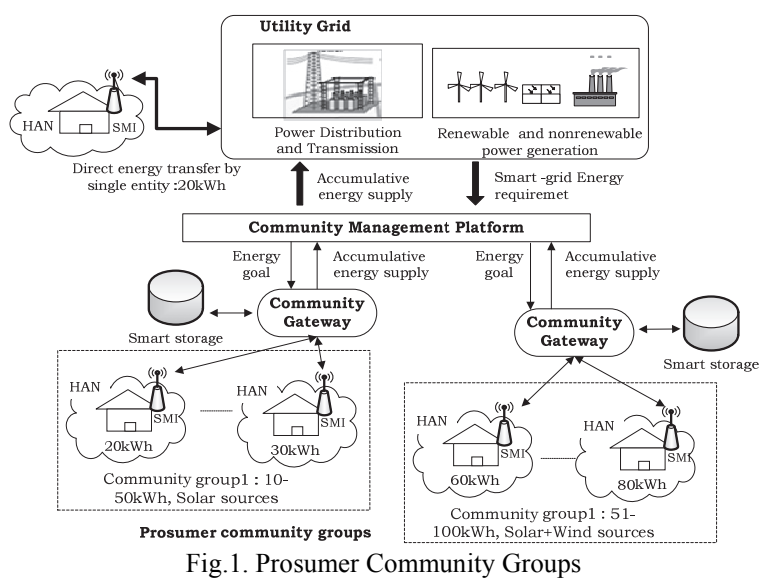

However, achieving a sustainable PCG involves addressing many socio-technical challenges such as formation, energy contribution assessment, incentive distribution etc. [9]. Nevertheless, no works have presented comprehensive suggestions to solve these challenges.

Therefore in this research paper, we address one of the key challenges of assessing the energy contribution of prosumers within a PCG to identify more 'influential prosumers', i.e. the prosumers who act favorably towards the nourishment of the community group.

We discuss the significance of addressing the aforementioned challenge from three perspectives as follows. First, from the community-management's perspective, this concept can be used to differentiate the more influential prosumers whose future behavior will facilitate the long-term sustainability of the community-group. Thus, the communitymanagement can take appropriate actions to make these influential prosumers feel privileged within the community group (by offering special incentives). Second, from the prosumers' perspective, being influential prosumers who generate and share higher amount of energy, they will receive special incentives (financial benefits and social responsibility). This will create a competitive environment among the prosumers, which will further encourage all the prosumers to invest in additional energy resources (e.g. putting more solar panels), knowing that the cost will be recovered within a specific time frame, and they will be getting greater profits as their energy generation capacity increases. Third, from the perspective of the society, the identification of influential prosumers, followed by higher incentives, motivates prosumers to generate green energy within their domestic environments, which is crucial to combat the problem of electricity shortage in the society, which is currently heavily dependent on non-renewable energy sources.

The rest of the article is arranged as follows; the literature of existing approaches on prosumer involvement in SG is presented in section II. In section III, we discuss the concept of PCG. The section IV presents the proposed methodology and section $\mathrm{V}$ highlights the verification of it. Finally the future directions and the concluding remarks are drawn in section VI.

\section{LITERATURE REVIEW}

In this section, we briefly analyze the background information of prosumer involvement in energy sharing process in existing smart grid projects. The two most common ways to involve the prosumers are; (i) Individual prosumer involvement, and (ii) Prosumer-group involvement.

\section{A. Individual prosumer involvement}

According to the existing literature (such as ADDRESS Project [11], S-Ten Project [12], and DR Project [13]), most of the energy-sharing approaches integrate and manage the prosumers as a single entity. This facilitates direct energy sharing between the prosumers and the utility grid and the energy-sharing decisions are made based on individual perceptions.

One of the apparent disadvantages of single-entity prosumer participation is that, the difficulty in receiving any real bargaining power when they sell low quantities of energy to the energy customers. Due to their perceived inefficiency and unreliability, such prosumers are often excluded from the energy market, thus unable to become regular and reliable supplier of energy to customers [7].

\section{B. Prosumer-group involvement}

Another method is to connect prosumer-groups to the utility-grid, where, a group of prosumers collectively sell the energy in energy market. This leads the prosumers to collectively attain higher mass of energy. The technical infrastructure of these prosumer groups can be identified either as VPPs (Virtual Power Plants) or micro-grids. A VPP $[14,15]$ is a large group of distributed energy resources (DERs) with an aggregated capacity, analogous to a usual power plant. Two VPP management architectures can be identified, namely centralized architecture and decentralized architecture. In the more common centralized architecture, grid-connected prosumers are controlled through a centralized controller. In contrast, in the decentralized VPP architecture, the energy sources are embedded with intelligent agents and perform independently in decision making.

On the other hand, the concept of microgrid [14]has been emerged as a localized grouping of electricity prosumers. The microgrids are smaller in size compared to the VPP and concerned with a locality in operation. One significant benefit of microgrids over VPPs is the reduced transaction cost as a result of a lesser number of intermediary parties [14]. 
However, aforesaid prosumer groups existing in literature are formed by merely bringing together a set of prosumers, without considering their diverse energy sharing behaviours (therefore, referred to as 'ad-hoc groups'). As a result, prosumers with extremely incompatible energy sharing behaviours could be found in the same group. This means that some prosumers might be sharing inadequate amounts of energy, thus reducing the overall performance of the entire group. This can discourage other prosumers in the same group, even causing some prosumers to leave the group, and ultimately resulting in an unsustainable prosumer groups in the long-term. In addition, the prosumers in these ad-hoc groups might be arbitrarily offering whatever amounts of energy they prefer, thus resulting in an unreliable energy supply to the energy buyers.

In order to address the shortcomings identified above, the concept of goal oriented PCG is proposed recently $[6,7,16]$, which we further analyze in Section III.

\section{GOAL ORIENTED PROSUMER COMMUNITY GROUPS}

The concept of Goal oriented PCG is an improved method of grouping prosumers [6, 7, 9], that is established by aggregating the prosumers having relatively similar energy behaviours. The prosumers of same PCG are located geographically closer for energy sharing among the local members, because energy transmission over long distances causes huge energy losses, and is also costly in operation.

Here, each prosumer community-group is associated with a prequalification criteria defined by the group, which a new prosumer should comply with in order to obtain its membership [10]. The two key parameters would be the upper energy boundary and the lower energy boundary of the PCG, which the prosumer's energy sharing capacity should lie in between to receive the membership of the corresponding group. The prosumers initiate a contract when they join the selected PCG.

The prosumers of a PCG can sell their unused energy to the prosumers of same PCG, who request energy in advance to meet the lower energy boundary as per the contract, or auction off their collective surplus energy to the external energy buying customers (e.g. utility grid). The prosumers receive standard tariff for the amount of shared energy and even the bonus incentives if they exceed the expectations (upper energy boundary) of the initial contract.

One of the key benefits of having PCGs is that the consumers can directly buy energy from geographically closer PCGs, rather than interrupting the main utility grid. In fact, the PCGs can fulfil the consumers' energy demand, in the longterm, in a more consistent manner than the individual prosumers or ad-hoc prosumer groups [10]. In addition, the PCGs would achieve a higher market visibility, hence would have a stronger bargaining power in the energy market in the long-term. The social impact of this advantage would lead to a more symmetrical interaction between a PCG and the large scale energy buyers (i.e., utility companies). In addition, compared to being an individual participant, being part of a bigger community group can also be seen as a big motivator for behavioural changes with regard to energy use practices, because the impact of a systematically formed PCG's behaviour can be more relevant and stronger than the impact of individual prosumer behaviours or ad-hoc group behaviours. As a result, PCGs can create a dynamic ecosystem of cooperating prosumers, and lead to a more sustainable and reliable energy sharing process.

However, the development of community based energy sharing network is still in its infancy, and hence the literature has paid very little attention on addressing the challenges involved. One such key challenge is finding influential prosumers within a PCG. Accordingly we illustrate the research problem we address in this research paper as follows.

- Problem Definition: How to rank the prosumers based on their short-term and long-term energy sharing behaviours, in order to find more influential prosumers in the PCG?

Even in a single PCG, the prosumers may exhibit dissimilar energy sharing behaviours over the membership duration. The two key dynamics that control the prosumers' energy sharing behaviours are their capacity of green energy generation and the household energy consumption. For instance, the domestic green energy generation is influenced by several factors, such as climate changes, malfunctioning or upgrade of renewable energy generation systems (e.g., solar systems), market influences, etc. [17]. Moreover, the household energy consumption is heavily dependent on the power loads, which are operated on the basis of regular and irregular energy requirements of different end-users. The operation of these power loads is sometimes seasonal (e.g., air conditioning in summer), and mostly unpredictable due to the unpredictable end-user demands and schedules. Such fluctuations in energy generation and consumption patterns can result in changing energy sharing behaviours by different users over a period of time. The energy sharing behaviours of 20 prosumers over time are illustrated in Fig.2 [17].

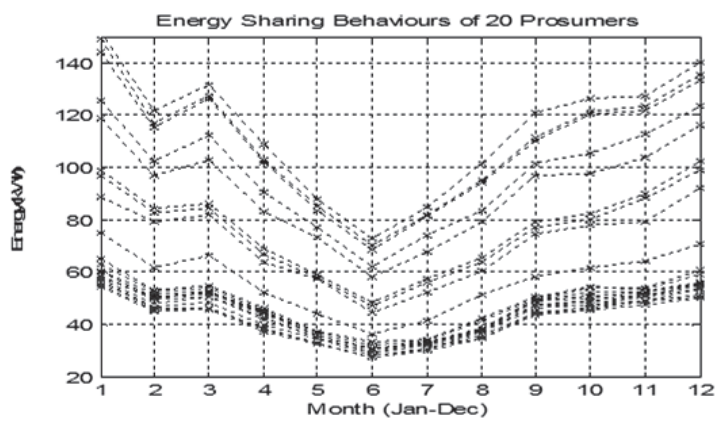

Fig.2. Energy sharing behaviors of 20 prosumers

Based on the diversity of the prosumers within a PCG, we identify a list of behaviours that a prosumer may demonstrate as follows;

- The prosumer supplies energy to meet the contract ( the supplied amount of energy lie within the upper and lower energy boundaries defined at the contract)

- The prosumer fails to meet the contract (i.e the lower energy boundary)

- The prosumer supplies more energy than the upper boundary of the contract

- The quality of the long term historic energy behaviours of 


\section{the prosumer}

- The prosumer shares energy with prosumers of local PCG, i.e. the prosumers' propensity to share the surplus energy with other local prosumers (in same PCG) who in need of energy to meet the lower energy boundary of the contract.

Due to such varied behaviours, treating all the prosumers in a PCG in the same fashion may be inequitable in certain scenarios like when distributing privileges (or incentives). This necessitates assessing and ranking the prosumers within a PCG to find out more influential prosumers. Therefore in the subsequent section, we propose a conceptual methodology for prosumer assessment and ranking.

\section{THE PROPOSED METHODOLOGY: PROSUMER ASSESSMENT AND RANKING}

In this section, we describe the proposed conceptual model for prosumer assessment and ranking within a PCG. The abstract view of the methodology is illustrated in Fig.3. In a PCG, the prosumers own different quantities of surplus energy. They can accumulate this surplus energy and sell to external customers like utility grid, and/or transfer the surplus energy to the local prosumers within the PCG. Accordingly, we make following assumptions and identify three main types of prosumers within the PCG.

i. Standalone prosumer: if the prosumer meets the lower energy boundary $\left(E_{\mathrm{lb}}\right)$ as required by the contract, but does not own surplus than the upper energy boundary $\left(E_{\mathrm{ub}}\right)$, that prosumer cannot buy or sell energy from fellow prosumers. The prosumer, who does not buy or sell energy from fellow prosumers, is called a "standalone prosumer".

ii. Energy buying prosumer: If the prosumer cannot meet $\mathrm{E}_{\mathrm{lb}}$, that prosumer can request energy from the fellow prosumers who have surplus energy than $E_{u b}$. We call such prosumer, who buys energy from fellow prosumers as "energy buying prosumer".

iii. Energy selling prosumer: If the prosumer owns surplus amount of energy than $\mathrm{E}_{\mathrm{ub}}$ as required by the contract, that prosumer is unable to buy the energy from other prosumers, but that prosumer can either sell the excessive energy to 'energy buying prosumers' or stay as a 'standalone prosumers'. The prosumer, who sells energy to local prosumers, is called an "energy selling prosumer".

As shown in Fig.3, the prosumer assessment and ranking scheme includes four on-going processes; (i) assessment criteria definition, (ii) criteria prioritization, (iii) point allocation, (iv) prosumer ranking. The 'assessment criteria definition' process involves definition of four assessment criteria, that respectively measure the prosumer's ability to meet the energy contract, to produce excessive energy than agreed, to share energy with the fellow prosumers, and their long-term historic energy sharing behaviours. In the second process of 'criteria prioritization', we prioritize the aforesaid criteria based on their importance in attaining PCG's enrichment. In the third process of 'point allocation', we

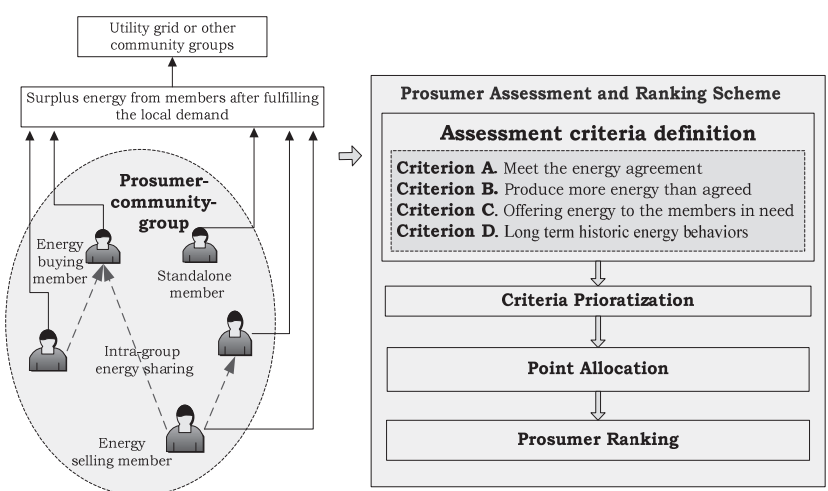

Fig.3. Conceptual methodology for prosumer assessment and ranking

allocate points to the prosumers based on their capacity in satisfying the criteria, and finally in the 'prosumer ranking' process, we rank the individual prosumers based on the allocated points.

In order to formulate an optimal solution for this methodology, one of the efficient theoretical platforms would be multiple-criteria decision-making techniques (MCDM) [18]. MCDM techniques have been successfully implemented in many application areas, like academic planning and health planning, and are claimed to be an efficient and feasible way to handle multiple conflicting criteria[18]. Nevertheless, this concept has not been used as a working model in energy networks. The techniques presented in MCDM have offered better results for ranking as compared to other well-known methods that present ranking based on the analysis of "Euclidean distance" or "Manhattan distance" among the objects[19]. Furthermore, the TOPSIS method (technique for order preference by similarity to ideal solution) of MCDM has shown better performance compared to the other methods in MCDM, such as "Max-min method", and "Min-max method" [19]. Therefore we use TOPSIS method of MCDM models to assess and rank a finite number of prosumers by measuring several conflicting criteria. In subsequent subsections, we discuss the theoretical formulation of the different processes of the methodology.

\section{A. Assessment criteria definition}

First of all, we decide the criteria which are used to assess the prosumers as follows; (i) Criterion A: Meet the energy agreement, (ii) Criterion B: Produce more energy than agreed, (iii) Criterion $\mathrm{C}$ : Offer energy to other prosumers in need (iv) Criterion D: Quality of the long-term historic energy behaviours.

\section{- Criterion A: Meet the energy agreement}

This criterion assesses the prosumer's ability to stay within the energy boundaries associated with the PCG. This is selected as an assessment criterion because, if a prosumer fails to meet the lowest energy boundary of the community-group, that prosumer is said to be in breach of the contract. The prosumers who lie within the boundaries of the community group are to be treated evenly with respect to this criterion.

\section{- Criterion B: Produce more energy than agreed}

This criterion assesses the prosumer's surplus energy sharing capacity, on top of the upper energy boundary of the community-group. This is selected as an assessment criterion 
because; the prosumers who offer higher amount of energy than the maximum expectation (upper energy boundary) can lead its PCG to receive incentives from main utility-grid and thus should be ranked higher than the usual prosumers.

\section{- Criterion C: Offer energy to other prosumers}

This criterion depends on the intra-PCG local energy sharing, where the prosumers having surplus energy than the upper energy boundary may share the energy with the local prosumers who fail to meet the lower energy boundary. In assessing this, we consider several factors; the amount of excess energy the energy selling prosumer is willing to sell, the energy requirement of the energy buying prosumer, the number of buyers in network and the amounts of energy a single buyer would buy from different sellers, and the amounts of energy a single seller would sell to different buyers. In this criterion, although the sharing energy among prosumers does not increase the collective energy of the PCG, it creates social sustenance within the PCG by minimizing the number of prosumers who may receive penalties due to the breach of contract. Therefore the prosumers who offer the energy to the fellow prosumers, who are in need of energy, should be treated favorably.

\section{- Criterion D: Quality of the long term historic energy behaviours}

All the above criteria (A, B, C) are measured as per the current time slot for the current 'transaction'. The term 'transaction' refers to the energy transfer to the external party (such as utility grid), after distributing the energy to the prosumers of the local community group. However, this criterion represents the quality of the long-term historic energy behaviours of a prosumer throughout the membership duration. In fact, it depends on how prosumers satisfy the criteria $\mathrm{A}, \mathrm{B}$, and $\mathrm{C}$ in all preceding timeslots. Due to the unpredictability involved in climate dependent renewable energy sources and prosumer household behaviours, the same prosumer who offers a lower amount of energy during this timeslot may have offered higher amount of energy throughout all the historic timeslots. Therefore, it is unjust to decide the prosumers' final ranks based only on their behaviours in the current timeslot. This fact necessitates the selection of this criterion.

\section{B. Criteria prioritization}

In this process, we prioritize the different assessment criteria adopting the methodology called "Weights from ranks" [18]. We prefer this weighting method ("Weights from ranks") over the other existing methods "Rating Method" and "Pair-wise Comparison" method due to following reasons. The Rating method provides a rating to each criterion, based on an agreed appropriate rating scale, and thus may fail to assure a ratioscale. On the other hand, the "Pair-wise Comparison" method (also called Ratio Weighing Method) compares two criteria at a time, using pair-wise evaluation. This method is not adaptable when the number of criteria is large because such methods require a large number of pair-wise comparisons, causing inconsistency inherent in a large number of such comparisons[18].
According to the "weights from ranks" method, the criterion weights can be obtained using equation 1 .

Let $r_{i}$ represent the position of the $i^{\text {th }}$ criterion. The criterion weight $\lambda_{i}$, for $k$ number of criteria given in equation 1 :

$$
\lambda_{i}=\frac{k-r_{i}+1}{\sum_{j=1}^{j=k}\left(k-r_{j}+1\right)}
$$

Accordingly, at first, the criteria are arranged in the order of increasing relative importance; the most important criterion takes the highest position in the criteria list. At this point we assign relative importance of criteria based on the organization's (community-management's) point of view as follows;

- Criterion A: This is the most important criterion, which highlights the prosumer's ability to meet the basic requirement of the PCG formation contract. This finds out whether the prosumers breach the contract or not. Therefore, this receives the highest position in the criteria list, thus receives highest criterion weight.

- Criterion B: Having surplus energy increases the overall collective energy of the community-group, resulting in receiving incentives from the utility grid. Therefore we assign this criterion the $2^{\text {nd }}$ highest position, hence receives second highest criterion weight.

- Criterion C and Criterion D: We assign both these criteria the $3^{\text {rd }}$ position. Although the criterions C and D do not make a direct impact on increasing the amount of overall surplus energy of the PCG for considered timeslot, these reveal the prosumer's aptitude to improve the longevity and sustainability of the PCG.

\section{Point allocation}

This process involves the allocating points to the prosumers according to their capability in satisfying the afore-identified criteria. We assign positive or negative points for the prosumers to represent the prosumers' contribution on each criterion as follows.

Ranking points for criterion $\mathrm{A}$ is proportional to the amount of energy shortage shown by a prosumer, who fails to meet the lowest energy boundary of the PCG, whereas it is zero for the others. Since showing an energy shortage adversely affects the performance of the PCG, the ranking points are in negative form. Let $E_{i}$ be the amount of energy presented by the prosumer, $E_{s h, i}$ is the energy shortage if any, $E_{l b}$ is the lowest energy boundary of the community-group. The $i^{\text {th }}$ prosumer's ranking points for criterion $\mathrm{A}\left(r p_{A, i}\right)$ is;

$$
r p_{A, i} \propto\left\{\begin{array}{cc}
-E_{s h, i} & ; \quad E_{i}<E_{l b} \\
0 & ; \quad E_{i} \geq E_{l b}
\end{array}\right.
$$

Similar to the above, ranking points for criterion B is proportional to the amount of surplus energy compared to the upper energy boundary, which takes a positive point form. This is zero for the prosumers, whose energy lies within the boundaries of the community group or who demonstrate energy shortage. Let $E_{i}$ be the amount of energy presented by the prosumer, $E_{e x, i}$ is the energy surplus if any, $E_{u b}$ is the upper energy boundary of the community-group. The $i^{t h}$ prosumer's 
ranking points for criterion $\mathrm{B}\left(r p_{B, i}\right)$ is;

$$
r p_{B, i} \propto\left\{\begin{array}{cl}
+E_{e x, i} & ; \quad E_{i}>E_{u b} \\
0 & ; \quad E_{i}<E_{u b}
\end{array}\right.
$$

The ranking points for criteria $\mathrm{C}$ is obtained by analyzing the prosumer's interactions with other prosumers within the community-group. We develop a method to find ranking points for criterion $\mathrm{C}$ by further developing some concepts of rank prestige and unidirectional graph edges [20]. In our method, the nodes are considered to be individual prosumers within the PCG. The edge is local energy trading that one prosumer would have with other prosumers. Let $\mathrm{N}_{\mathrm{b}}$ is the number of energy buying prosumers; $\mathrm{N}_{\mathrm{s}}$ is the number of energy selling prosumers; $\mathrm{N}$ is total number of prosumers in the community-group. The main measurements of assessing criterion $\mathrm{C}$ are, $\mathrm{E}_{\mathrm{i}, \rightarrow \mathrm{q}}$, that denotes the amount of energy sold by $\mathrm{i}^{\text {th }}$ energy selling prosumer to $\mathrm{q}^{\text {th }}$ prosumer and $\mathrm{E}_{\mathrm{i}}<\mathrm{p}$, that denotes the amount of energy bought by $i^{\text {th }}$ energy buying prosumer from $\mathrm{p}^{\text {th }}$ prosumer. The ranking points of criterion C for three different prosumer prosumer types; energy selling prosumers, energy buying prosumers, and standalone prosumers are as follows;

$$
r p_{C, i} \propto\left\{\begin{array}{c}
\sum_{q=1}^{N b} E_{i \rightarrow q} \times \frac{N_{b}}{N} ; \text { for all } i \text { (energy selling prosumers) } \\
\sum_{p=1}^{N s} E_{i \leftarrow p} \times \frac{N_{s}}{N} ; \text { for all } i \text { ( energy buying prosumers) } \\
0 \quad ; \text { for standalone prosumers }
\end{array}\right.
$$

The ranking points for criterion $\mathrm{D}$ depends on how the prosumer behaves in historic timeslots and is proportional to the combined ranking points in all the historic timeslots throughout the membership record. Let $n$ be the considered time slot, $R P_{i, h}$ be the accumulated ranking points a prosumer obtained in historic timeslots. The ranking points a prosumer obtained for criterion $\mathrm{D}\left(r p_{D, i}\right)$ is given by;

$$
r p_{D, i} \propto R P_{i, h} ; \text { for all } n>2
$$

\section{Prosumer ranking}

This process involves the ranking of the prosumers of the PCGs. The TOPSIS method, which we adopted here for ranking is explained as follows; Let $x$ is any prosumer within the single PCG and $C 1(x), C 2(x), C 3(x)$, and $C 4(x)$ are the expected values for four evaluation criteria. The objective of this model is to rank the participating prosumers of a PCG based on their ability to meet the expected level of achievement of criteria values or 'maximize [Cl(x), $C 2(x)$, $C 3(x), C 4(x)]$ '. Thus, the chosen attribute of the prosumer is compared with the "ideal solution", $H^{*}$, which is the highest expectation for that criterion $j$, and the "negative-ideal solution", $L^{*}$, which is the lower bound of the same criterion $j$. This method produces an index combining these factors and the prosumer that maximizes this index value by showing closest result to "ideal solution" and farthest from the "negative-ideal solution", is ranked $1^{\text {st }}$.

The theoretical adaptation of the model can be explained as follows; We first define the normalized pay-off matrix $\left(r_{i j}\right)$ for four criteria as;

$$
r_{i j}=\frac{r p_{j i}}{\left[\Sigma_{\mathrm{i}}\left(r p_{j i}\right)^{2}\right]^{1 / 2}} ; 1 \leq i \leq N ; j=1,2,3,4
$$

Where, $r p_{j i}$ is the ranking points for $i^{\text {th }}$ prosumer for $j^{\text {th }}$ evaluation criterion. $N$ is number of prosumers within the group.

The weighted pay-off matrix, $q_{i j}$, is computed using the importance weights obtained by Equation (1).

$q_{i j}=\lambda_{j} \times r_{i j}$

where, $\lambda_{j}$ is the relative importance weight of the $j^{\text {th }}$ criterion

Using the weighted pay-off matrix, ideal solution, $H_{j}^{*}$ is defined as the maximum of $q_{i j}$, for all $i$ and $j=1,2,3,4$; and the anti-ideal solution, $L^{*}$ as the minimum of $q_{i j}$, for all $i$ and $j=1,2,3,4$. Accordingly, the separation measures with ideal solution and anti-ideal solution for each prosumer are calculated.

Separation measure of $i^{\text {th }}$ prosumer's performance for $j^{\text {th }}$ criterion with ideal solution $\left(D_{H i}\right)$ is given in Equation 8;

$\mathrm{D}_{\mathrm{Hi}}=\left[\sum_{j}\left(q_{i j}-H_{j}^{*}\right)^{2}\right]^{1 / 2}$

Separation measure of $i^{\text {th }}$ prosumer's performance with antiideal solution $\left(D_{L i}\right)$ is given in Equation 9;

$\mathrm{D}_{\mathrm{Li}}=\left[\sum_{j}\left(q_{i j}-L_{j}^{*}\right)^{2}\right]^{1 / 2}$

These two measures are combined to develop a ranking index, " $S$ " as given in the equation 10 . A prosumer showing a higher index value is in higher rank.

$\mathrm{S}=\frac{D_{L i}}{D_{L i}+D_{H i}} ; i=1,2,3, \ldots, N$

Here $0 \leq S \leq 1 ; S=0$ when the performance of $i^{\text {th }}$ prosumer is same as the anti-ideal solution and $S=1$ when the performance of $i^{\text {th }}$ prosumer is the ideal solution.

\section{RESUltS AND Discussion}

In this section, we verify and validate the functionality of the prosumer ranking methodology. The dataset we have used here was synthetically generated dataset following the realistic energy consumption, generation and sharing models in Australian conditions. In this simulation, we define the attributes of the selected PCG as follows: the defined lower energy boundary of the community group is $50 \mathrm{kWh}$ per month and the defined upper energy boundary of the community-group is $150 \mathrm{kWh}$ per month. Here, $50 \mathrm{kWh}$ and $150 \mathrm{kWh}$ are the minimum and the maximum figures of averaged surplus energy shown by an energy user during the summer months (December-February) in Australian conditions, i.e., the difference between the amount of energy generated by $3 \mathrm{kWh}$ solar energy systems and the quantity of energy consumed by average consumer [17]. Further information about this dataset can be found in our previous work[17]. For the purposes of clarity, in this paper we consider only 10 prosumers in the selected PCG, and two 
timeslots (the very first timeslot after the formation of the PCG, and the subsequent timeslot). However, this methodology can be utilized for any number of prosumers and any number of timeslots. Moreover, in order to evaluate the Criterion C, we introduce energy buying prosumers", "energy selling prosumers" and "standalone prosumers" to our dataset.

For the considered dataset, we illustrate the guidelines of four criterions as follows;

- Criterion A: the ranking points are proportional to the energy shortage calculated from 50kWh.

- Criterion B: the ranking points are proportional to the surplus energy calculated from $150 \mathrm{kWh}$.

- Criterion C: prosumers who fail to meet $50 \mathrm{kWh}$ can buy energy from other prosumers until they reach $50 \mathrm{kWh}$ (lower energy boundary). The energy can be bought only from the prosumers, who own surplus energy than 150 $\mathrm{kWh}$. Ranking points are proportional to the positive value of energy given to the other prosumers and negative value of the energy bought from other prosumers.

- Criterion D: evaluation of Criterion D adds iterative nature to the final ranking points of a prosumer. In this simulation, we consider two timeslots to show the effect of criterion D. For the $1^{\text {st }}$ timeslot, the prosumer ranks do not depend on the criterion $\mathrm{D}$, because, as being the very first timeslot after the community group formation, previous timeslots do not exist. For the $2^{\text {nd }}$ timeslot, the ranks are influenced by the energy behaviors of the $1^{\text {st }}$ timeslot.

As the first criterion is the most important criterion from all four, it receives the highest importance weight calculated via the equation 1 , which is 0.364 . The $2^{\text {nd }}$ criterion receives the second highest priority with importance weight of 0.272 . Finally the $3^{\text {rd }}$ and $4^{\text {th }}$ criterions receive the importance weights of 0.182 .

Table. 1 and Table. 2 respectively illustrate the necessary measurements for four criteria for $1^{\text {st }}$ timeslot and $2^{\text {nd }}$ timeslot and the final ranks of prosumers obtained using the proposed methodology. The process of ranking follows the equations (2)-(10), and the final ranks of the prosumers are decided based on the ranking index (" $S$ " value of Equation (10)).

Fig.4 and Fig.5 demonstrate the prosumers' final ranks obtained for each time slot. Here we compare the ranks obtained via the proposed ranking scheme (Table I and II) with the conventional ranking that ranks the prosumers based on the distance measures (Manhattan distance) in single dimension (energy owned). According to the Fig.4, with conventional ranking method, the $4^{\text {th }}$ and $5^{\text {th }}$ prosumers have received the same rank based on the same amount of energy they own, but with the proposed ranking method, the $4^{\text {th }}$ prosumer receives better rank as that prosumer shares higher percentage of excess energy of $10 \mathrm{kWh}$ with the other prosumers who in need, compared to $5^{\text {th }}$ prosumer, who only shares $5 \mathrm{kWh}$ with other prosumers. The effect of the criterion $\mathrm{D}$ is clearly visible in Fig.5, in which the $6^{\text {th }}$ and $7^{\text {th }}$ prosumers, who own similar amount of energy, are ranked same in conventional ranking method. However in the proposed ranking method, the $7^{\text {th }}$ prosumer receives better rank than the $6^{\text {th }}$, as the $7^{\text {th }}$ prosumer shows comparatively better performances in the previous timeslot.

TABLE I

ENERGY SHARING PATTERNS OF 10 PROSUMER COMMUNITY-GROUP PROSUMERS IN 1ST TIMESLOT

\begin{tabular}{ccccccc}
\hline \hline $\begin{array}{l}\text { Pros } \\
\text { umer } \\
\text { ID }\end{array}$ & $\begin{array}{c}\text { Energy } \\
\text { Owned } \\
(\mathrm{kWh})\end{array}$ & $\begin{array}{c}\text { criterio } \\
\mathrm{n} \mathrm{A} \\
(\mathrm{kWh})\end{array}$ & $\begin{array}{c}\text { criterion } \\
\mathrm{B}(\mathrm{kWh})\end{array}$ & $\begin{array}{c}\text { criterio } \\
\mathrm{n} \mathrm{C} \\
(\mathrm{kWh})\end{array}$ & $\begin{array}{l}\text { criter } \\
\text { ion } \\
\mathrm{D}\end{array}$ & $\begin{array}{c}\text { Final } \\
\text { rank }\end{array}$ \\
\hline 1 & 50 & 1 & 0 & 0 & $\mathrm{NA}$ & 5 \\
2 & 46 & -6 & 0 & -14 & $\mathrm{NA}$ & 8 \\
3 & 190 & 1 & 40 & 20 & $\mathrm{NA}$ & 1 \\
4 & 176 & 1 & 26 & 10 & $\mathrm{NA}$ & 2 \\
5 & 176 & 1 & 26 & 5 & $\mathrm{NA}$ & 3 \\
6 & 36 & -14 & 0 & -14 & $\mathrm{NA}$ & 8 \\
7 & 143 & 1 & 0 & 0 & $\mathrm{NA}$ & 5 \\
8 & 100 & 1 & 0 & 16 & $\mathrm{NA}$ & 4 \\
9 & 70 & 1 & 0 & 0 & $\mathrm{NA}$ & 5 \\
10 & 28 & -22 & 0 & -22 & $\mathrm{NA}$ & 10 \\
& & & & & &
\end{tabular}

TABLE II

ENERGY SHARING PATTERNS OF SAME 10 PROSUMER COMMUNITY-GROUP PROSUMERS IN 2ND TIMESLOT

\begin{tabular}{|c|c|c|c|c|c|c|}
\hline $\begin{array}{c}\text { Prosum } \\
\text { er ID }\end{array}$ & $\begin{array}{c}\text { Energy } \\
\text { Owned } \\
(\mathrm{kWh})\end{array}$ & $\begin{array}{c}\text { criteri } \\
\text { on A } \\
(\mathrm{kWh} \\
)\end{array}$ & $\begin{array}{c}\text { criterio } \\
\text { n B } \\
(\mathrm{kWh})\end{array}$ & $\begin{array}{l}\text { criterion } \\
\mathrm{C}(\mathrm{kWh})\end{array}$ & $\begin{array}{c}\text { criterio } \\
\text { n D }\end{array}$ & $\begin{array}{l}\text { Final } \\
\text { Rank }\end{array}$ \\
\hline 1 & 38 & -12 & 0 & -12 & 5 & 10 \\
\hline 2 & 184 & 0 & 34 & 34 & 8 & 1 \\
\hline 3 & 45 & -5 & 0 & -5 & 1 & 8 \\
\hline 4 & 40 & -10 & 0 & -10 & 2 & 9 \\
\hline 5 & 188 & 0 & 38 & 10 & 3 & 2 \\
\hline 6 & 100 & 0 & 0 & 0 & 8 & 7 \\
\hline 7 & 100 & 0 & 0 & 0 & 5 & 6 \\
\hline 8 & 100 & 0 & 0 & 0 & 4 & 5 \\
\hline 9 & 164 & 0 & 14 & 12 & 5 & 3 \\
\hline 10 & 158 & 0 & 8 & 8 & 10 & 4 \\
\hline
\end{tabular}

First timeslot - Ranking based on conventional method (according to the amount of energy owned) vs. proposed method

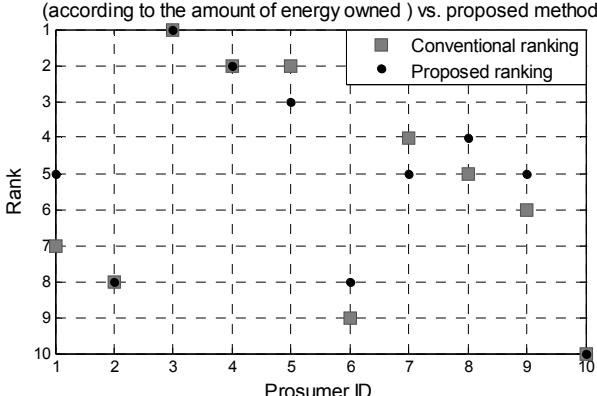

Fig. 4. Ranks of prosumers in $1^{\text {st }}$ timeslot

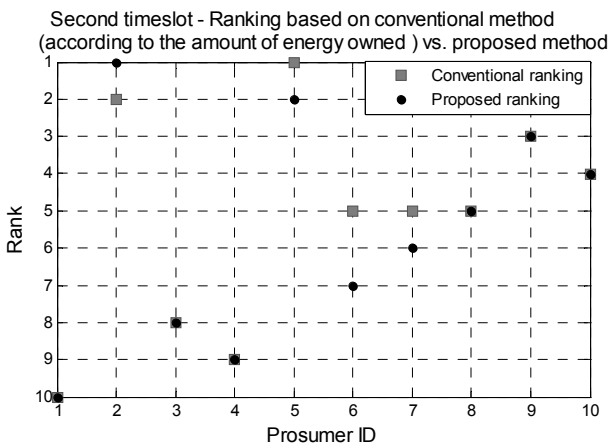

Fig. 5. Ranks of prosumers in $2^{\text {nd }}$ timeslot 
Overall, the proposed method that coordinates different criteria shows a fairer ranking than the conventional ranking methods that consider a single dimension. Moreover, we discuss the adaptability of this methodology as follows. Here, we take the term "adaptable" in the sense of the system being adaptable to adverse weather conditions. For instance, we use following scenario: suppose an unfavorable weather is forecasted for a future timeslot that would prevent the prosumers' green energy generation. In that case, the organization may request the prosumers to supply energy to a special energy storage, which could be released during the upcoming timeslot that is forecasted with an adverse weather condition. In such a scenario, the prosumer assessment and ranking methodology can be further developed by adding a new assessment criterion to assess the quantity of energy supplied by the prosumers to the special energy storage unit, and accordingly rank the prosumers.

\section{CONCLUSION AND FUTURE WORK}

In this paper, we addressed the challenge of prosumer assessment and ranking in PCGs in SG. The suggested methodology offered an effective ranking when dealt with multiple assessment criteria. In future, we hope to experiment this technique to a large dataset of prosumers in actual environment. Furthermore, we plan to investigate other related challenges involved in making sustainable PCGs.

\section{REFERENCES}

World Energy Demand and Economic Outlook Available: http://www.eia.doe.gov/oiaf/ieo/world.html

V. C. Gungor, D. Sahin, T. Kocak, S. Ergut, C. Buccella, C. Cecati, and G. P. Hancke, "Smart Grid Technologies: Communication Technologies and Standards," IEEE Transactions on Industrial Informatics, vol. 7, pp. 529-539, 2011.

P. Siano, C. Cecati, H. Yu, and J. Kolbusz, "Real Time Operation of Smart Grids via FCN Networks and Optimal Power Flow," IEEE Transactions on Industrial Informatics, vol. PP, pp. 1-1, 2012.

Y. Xu, Z. Dong, Z. Xu, K. Meng, and K. Wong, "An Intelligent Dynamic Security Assessment Framework for Power Systems with Wind Power," IEEE Transactions on Industrial Informatics, vol. PP, pp. 1-1, 2012.

Y. Xinghuo, C. Cecati, T. Dillon, Simo, x, and M. G. es, "The New Frontier of Smart Grids," Industrial Electronics Magazine, IEEE, vol. 5, pp. 49-63, 2011.

A. J. D. Rathnayaka, V. M. Potdar, O. Hussain, and T. Dillon, "Identifying prosumer's energy sharing behaviours for forming optimal prosumer-communities," presented at the International Conference on Cloud and Service Computing (CSC), 2011.

A. J. D. Rathnayaka, V. M. Potdar, and S. J. Kuruppu, "An innovative approach to manage prosumers in Smart Grid," presented at the World Congress on Sustainable Technologies (WCST), 2011.

A. J. D. Rathnayaka, V. M. Potdar, and S. J. Kuruppu, "Evaluation of wireless home automation technologies," in Digital Ecosystems and Technologies Conference (DEST), 2011 Proceedings of the 5th IEEE International Conference on, 2011, pp. 76-81.

A. J. D. Rathnayaka, V. M. Potdar, and S. J. Kuruppu, "Design of Smart Grid Prosumer Communities via Online Social Networking Communities," International Journal of Infornomics, vol. 5, 2012. A. J. D. Rathnayaka, V. M. Potdar, T. Dillon, and O. Hussain, "An innovative Approach to Form prosumer Community Groups in Smart Grid," Energy Policy Journal, 2013.

R. Belhomme, R. C. R. D. Asua, G. Valtorta, A. Paice, F. Bouffard, R. Rooth, and A. Losi, "ADDRESS - active demand for the smart grids of the future," IET Seminar Digests, vol. 2008, p. 33, 2008

[12] S-TEN Project: Intelligent Self-describing Technical and Environmental Networks. Available: http://www.s-ten.eu/

[13] Demand Response Project. Available: http://www.ieso.ca/imoweb/consult/demandresponse.asp

[14] P. Asmus, "Microgrids, Virtual Power Plants and Our Distributed Energy Future," The Electricity Journal, vol. 23, pp. 72-82, 2010.

[15] G. Chalkiadakis, V. Robu, R. Kota, A. Rogers, and N. Jennings, "Cooperatives of Distributed Energy Resources for Efficient Virtual Power Plants," presented at the The Tenth International Conference on Autonomous Agents and Multiagent Systems (AAMAS-2011), Taipei, Taiwan, 2011

[16] S. Karnouskos, "Communityware Smartgrid," presented at the 21st International Conference on Electricity Distribution, Frankfurt, 2011.

[17] A. J. D. Rathnayaka, V. M. Potdar, O. Hussain, and T. Dillon, "Analysis of Energy Behaviour Profiles of Prosumers," in IEEE International Conference on Industrial Informatics, 2012, pp. 236 $-241$.

[18] A. S. M. Masud and A. R. Ravindran, "Multiple Criteria Decision Making," in Operations Research Methodologies, ed: Taylor \& Francis Group, LLC, 2009.

[19] R. Zia ur, "Iaas Cloud Selection using MCDM Methods," 2012, pp. 246-251.

[20] H. W. Lam and W. Chen, "Finding Influential eBay Buyers for Viral Marketing A Conceptual Model of BuyerRank," presented at the International Conference on Advanced Information Networking and Applications, 2009.

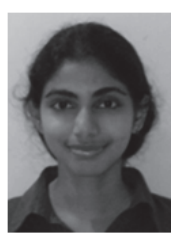

A J Dinusha Rathnayaka graduated with an honours degree in Electronics and Telecommunication Engineering from the University of Moratuwa, Sri Lanka, and currently reading for $\mathrm{PhD}$ at School of Information Systems, Curtin University Australia. She has published more than 20 publications in journals and conferences. Her main areas of research include smart grids, wireless sensor networks, and data mining.

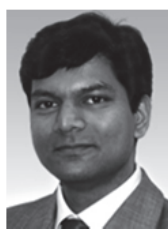

Vidyasagar M. Potdar graduated from the Gujarat University, India, and received Masters from University of Newcastle, Australia and $\mathrm{PhD}$ from Curtin University Australia. He is currently working in School of Information Systems in Curtin University as a SENIOR RESEARCH FELLOW. He has published more than 100 publications in top journals and conferences and his $\mathrm{H}$ index is 16 . His main areas of research include smart grids, wireless sensor networks \& information security.

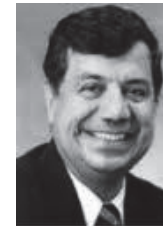

Tharam S. Dillon (M'74-SM87-F'98-LF'10) is the RESEARCH PROFESSOR at Curtin University, Australia. $\mathrm{He}$ has published more than 750 papers published in international conferences and journals and is the author of five books and has another five edited books. His current research interests include smart grid, web semantics, ontologies, internet computing, e-commerce, neural nets, software engineering, database systems, and data mining.

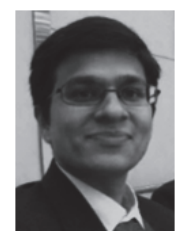

Omar K. Hussain is a RESEARCH FELLOW at Curtin University, Australia. He has published more than 35 papers published in international conferences and journals His current research interests include Smart grid, Risk assessment and management in business decision making, business intelligence, computational intelligence, and cloud computing.

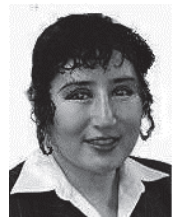

Elizabeth Chang (M'02-SM'07) is a RESEARCH PROFESSOR in Curtin University, Australia. She has coauthored three books and has published over 350 scientific papers. Some of her research interest includes: smart-grids, ontology and multi-agent systems, data mining, XML, Web services and IT for business and commerce. 